1 Osame M, Usuku K, Izumo S, et al. HTLV-I associated myelopathy, a new clinical entity. associated myelopathy,

2 Dalakas MC, Engel WK. Chronic relapsing (dysimmune) polyneuropathy: pathogenesis and treatment. Ann Neurol 1981;9(suppl): $134-45$.

3 Tourtollote $W$, Staugaitis S, Walsh, et al. The basis of intra-blood-brain-barrier IgG synthesis. Ann Neurol 1985;17:21-7.

4 Cornblath DR, MaCarthur JC, Kennedy PGE et al. Inflammatory demyelinating periphera neuropathies associated with human $\mathrm{T}$-cell lymphotropic virus type III infection. $A n n$ Neurol 1987;21:32-40.

5 Tsukada $M$, Chang-Sung $K$, Inoue $A$, et al. Demyelinating neuropathy associated with hepatitis B virus infection: detection of immune complexes composed of hepatitis B
virus surface antigen $J$ Neurolog Sci 1987; virus surfo3-16.

\section{Carotid gustatory syndrome in a patient with Holmes-Adie syndrome}

Holmes-Adie syndrome comprises the "tonic pupil" and reduced or absent deep tendon reflexes, ${ }^{1-3}$ and it can be accompanied by various other symptoms such as segmental hypohidrosis, impaired sudomotor and vasomotor reflexes, cardiac autonomic dysfunction, orthostatic hypotension and peripheral neuropathy. We report a patient with Holmes-Adie syndrome who noticed an unusual taste sensation in the left posterior part of the tongue when the neck was pressed on the same side. The patient was a 57 year old Japanese woman who was found to have dilated and non-reactive pupil in the left eye. Shortly after, she noticed that pressing on the left anterior neck elicited a "metallic" taste on the left posterior part of her tongue. Otherwise she was asymptomatic.

General examination was normal. The left pupil was irregularly shaped and $6 \mathrm{~mm}$ in diameter, while the right pupil was $4 \mathrm{~mm}$ in diameter. The left pupil reacted to light very slowly and incompletely to a minimal diameter of $4 \mathrm{~mm}$ while the right pupil reacted promptly to $2 \mathrm{~mm}$. In a dark room, the right pupil dilated promptly, but the left pupil was very sluggish in dilatation. Convergence reflex was moderately slow in the left while it was normal in the right. Accommodation was normal with the near point being $10 \mathrm{~cm}$ bilaterally. The left pupil showed an excessive response to the application of $2.5 \%$ methacholine and became much smaller than the right. Visual acuity, visual field and ocular fundi were normal. There was no extraocular muscle palsy. Facial muscles, facial sensation and corneal reflexes were all normal, as was her hearing. Soft palate and tongue were normal. Lacrymation and salivation were normal.

Pressure on the left anterior neck overlying the carotid sinus immediately produced a "metallic" taste on the left posterior tongue. Heart rate on ECG was slightly decreased by the carotid pressure. Pressing the right side of the neck did not produce any gustatory sensation. Superficial sensation of the tongue and pharynx was normal, and taste sensation was normal over the tongue. Cranial nerves were otherwise normal. There were no motor or sensory abnormalities in the limbs except for loss of ankle reflexes bilaterally even with reinforcement. The skin was moderately moist. There was no sphincter impairment. The supine BP of $122 / 88$ fell to $96 / 0$ on standing although the patient did not com- plain of any dizziness and the pulse rate did not change.

Laboratory tests including complete blood count, blood sugar, ESR, CRP, serum protein fraction, RA test, anti-nuclear antibody, anti-DNA antibody, anti-RNP antibody, LE tests and ECG were all normal or negative. During hyperventilation, the heart rate increased from 66 to 96 beats per minute. Needle EMG showed no abnormality. The conduction velocity of the posterior tibial nerve and the sural nerve was 43 and $44 \mathrm{~ms}$, respectively. The distal motor latency of the right posterior tibial nerve was $5.1 \mathrm{~ms}$ with the amplitude of the evoked EMG being $5 \mathrm{mV}$. Neither $\mathrm{H}$-reflex nor Treflex could be elicited in the gastrocnemius muscles.

The diagnosis of Holmes-Adie syndrome was made based on the presence of a tonic pupil associated with overactive response to methacholine and the absence of ankle jerks which was proved by $\mathrm{H}$-reflex study. There were at least two more features in the present case: orthostatic hypotension and a unique syndrome consisting of gustatory sensation elicited by pressure applied to the carotid sinus of the same side. Johnson $e^{4}$ al $^{4}$ reported two patients with Holmes-Adie syndrome accompanied by orthostatic hypotension. Their cases were found to have afferent block from baroreceptors in contrast to the efferent autonomic block found in most other cases of idiopathic orthostatic hypotension. In our case, it is less likely that the orthostatic hypotension was due to the afferent baroreceptor block because the heart rate responded normally to the carotid massage.

The most unusual feature in our case was the "metallic" taste sensation elicited in the left posterior part of the tongue by pressing the anterior neck on the same side. To our knowledge this phenomenon has not been described previously. The gustatory sensation elicited by the carotid pressure can be best explained by postulating a misconnection, within the glossopharyngeal nerve, of the afferent impulses from carotid baroreceptors with the sensory impulse from taste fibres originating from the posterior third of the tongue. This condition may be called "carotid gustatory syndrome". Pathogenesis of Holmes-Adie syndrome varies from case to case, and it may be associated with collagen disease, especially systemic lupus erythematosus. In our case, however, we could not demonstrate any underlying cause. In view of the association with orthostatic hypotension and unilateral glossopharyngeal neuropathy, it is most likely to be a part of systemic poly- and multiple neuropathy involving autonomic and somatic peripheral nervous systems.

\section{HIROSHI SHIBASAKI} HIDEAKI TOMI* National Institute of Neuroscience, and Division of Neurology,
National Centre of Neurology and Psychiatry, Kodaira, Tokyo, 187 Japan

1 Adie WJ. Argyll Robertson pupils, true and false. Br Med J 1931;2:136-8

2 Adie WJ. Tonic pupil and absent tendon reflexes, a benign disorder sui generis: its complete and incomplete forms. Brain 1932; 55:98-113.

3 Holmes G. Partial iridoplegia associated with symptoms of other disease of the nervous system. Trans Ophthalmol Soc (UK) 1931; 51:209-28.

4 Johnson RH, McLelan DL, Love DR. Orthostatic hypotension and the Holmes-Adie syndrome. A study of two patients with
afferent baroreceptor block. $J$ Neurol Neurosurg Psychiatry 1971;34:562-70.
The relationship of memory and cognition in Parkinson's disease to lateralisation of motor symptoms

A recent report' indicated that Parkinson's disease (PD) patients with greater involvement on the left body side were more impaired than right body patients in memory, visuospatial performance, language and mental control. A question was later raised as to whether this was due to actual lateralised differences or more a function of severity of disease and disability. ${ }^{2}$ In view of the apparent contradiction we explored other possible left-right hemisphere differences in psychological functions in the hemi-Parkinsonian patients to determine further the role of symptom severity and disability in such differences.

Fifty three consecutively tested patients with idiopathic PD served as the pool of subjects. All were outpatients receiving Sinemet treatment and almost half were receiving Symmetrel as well. The mean age of the group was 68 years (range 41-83). There were 21 females and 32 males. Mean length of formal education was 12.3 years (range 5 to 20 years). Mean length of illness was 12 years, five months (range three months to 57 years) Pattern and degree of symptoms and functional status varied widely. To secure other pertinent data, charts were reviewed for marital status, age, education, sex, length of illness and age at onset of illness. Dosage and time on medication for Sinemet and Symmetrel were also tabulated. For symptom and disability factors, clinical ratings were available for tremor, rigidity, alternating movements, bradykinesia and functional activities.

It was then determined which side of the body showed greater involvement by totalling the ratings for tremor, rigidity and alternating movement impairment for each side. If the total score was one rating point or more different between sides, the side with the higher score was deemed primarily involved. On this basis, 15 left side and 10 right side dominant patients were identified. For the left side dominant group the total ratings ranged from 0 to 8 ( $\mathrm{L}$ mean $=5.9 ; \mathrm{R}$ mean $=3.0, t<0.01)$. For the right side dominant group they ranged from 0 to 7 (L mean $=2.2 ; R$ mean $=4.9, t<0.01$ ). The groups are significantly different for the side of the body most affected.

To determine the total symptom severity for each patient, ratings of bradykinesia and impaired function were added to those of tremor and rigidity

A modification of the Randt Memory Test ${ }^{3}$ was used to evaluate memory functions. Several aspects of episodic memory were evaluated, including rote, associative, discourse and visual recognition. The modules used were general information, recall of five items, recall of a short story and recall of drawings of everyday objects. Cognitive functions were assessed by administration of the following subtests of the Wechsler Adult Intelligence Scale-Revised: ${ }^{4}$ Information, Digit Span, Vocabulary, Similarities, Picture Completion, Block Design and the Digit Symbol. The battery includes both verbal and non-verbal tests. Prorated Verbal and Performance IQ scores were also derived.

The left and right side dominant groups did not differ for age, age at onset, sex, length of illness, education, or length of time on and dosage of medication. They did not differ in total tremor, rigidity or alternative movement impairment, but did differ significantly in 